Original Article (short paper)

\title{
Sodium bicarbonate supplementation in resistance exercise performance, perceived exertion and blood lactate concentration
}

\author{
Letícia Rodrigues Fontanella $^{1}$ (D), Cíntia Azara ${ }^{1}$ (D), Estevão Scudese ${ }^{2,3}$ (D), \\ Diogo de Oliveira Silva ${ }^{2}$ (D), Carlos José Nogueira ${ }^{3}$ (D), Michelle Soraia Espínola Costa ${ }^{4}$ (D), \\ Gilmar Weber Senna ${ }^{2,3}$ \\ ${ }^{1}$ Colégio Arthur Sá Earp Neto, Departamento de Nutrição, Petrópolis, RJ, Brasil. \\ ${ }^{2}$ Universidade Católica de Petrópolis, Laboratório de Ciências do Esporte e Exercício, \\ Petrópolis, RJ, Brasil. \\ ${ }^{3}$ Universidade Federal do Estado do Rio de Janeiro, Laboratório de Biociências da Motricidade \\ Humana, Rio de Janeiro, RJ, Brasil. \\ ${ }^{4}$ Universidade Federal do Estado do Rio de Janeiro, Programa de Pós-graduação em Infecção \\ HIV/AIDS e Hepatites Virais, Rio de Janeiro, RJ, Brasil.
}

\begin{abstract}
Aim: The aim was to evaluate the effect of sodium bicarbonate supplementation $\left(\mathrm{NaHCO}_{3}\right)$ in repetition performance, perceived exertion and blood lactate concentration. Methods: Fourteen trained men (25.14 \pm 3.5 years; $85.83 \pm 10.18 \mathrm{~kg} ; 1.78 \pm 0.06 \mathrm{~m} ; 26.88 \pm 3.17 \mathrm{~kg} / \mathrm{m}^{2}$ ) realized two upper-body resistance exercise sessions consisting of 15-RM load and 1 minute of the interval, combining bicarbonate supplementation (BS) or Placebo conditions. Results: After the ANOVA analysis, no difference was found on the total number of repetitions during the session on both BS and Placebo condition $(\mathrm{p}=0.11)$. However, a greater number of total of repetitions on the machine chest fly exercise for BS vs. Placebo condition $(\mathrm{p}=0.04)$ was observed. The perceived exertion increased regardless of the supplementation condition $(\mathrm{p}<0.0001)$. Additionally, for the blood lactate was observed significant increases in BS vs. Placebo condition in the post verification $(\mathrm{p}=0.013)$, corroborated for a bigger area under the curve (AUC) on the BS compared to Placebo $(p=0.026)$ condition. Conclusion: In conclusion, sodium bicarbonate supplementation was not able to improve performance during a resistance exercise session, except for the single-joint exercise performed with high values of perceived exertion, not altering the perceived exertion, and blood lactate between protocols.
\end{abstract}

Keywords: muscle strength, physical fitness, alimentary supplementation, sodium bicarbonate.

\section{Introduction}

According to the American College of Sports and Medicine $\mathrm{ACSM}^{1}$, on the most recent position statement regarding nutrition and athletic performance, sodium bicarbonate $(\mathrm{NaHCO})$ has been used as an ergogenic resource. This practice is often applied to improve performance and reduce the fatigue on high-intensity exercises, mainly on interval and intermittent exercises (like multiple sprints). Some evidence has shown that the improvement in performance in an interval and intermittent exercise methods are related to optimization on acid/base imbalances as an outcome of the energy expenditure reactions on high-intensity exercises ${ }^{2-8}$.

The positive physiological effect of the $\mathrm{NaHCO} 3$ supplementation appears to be acute, peaking around 30 to 90 minutes after its ingestion, by enhancing the extracellular buffering capacity ${ }^{1,2}$. An explanation for the performance enhancement during exercise is that the buffer induced by $\mathrm{NaHCO} 3$ increases the glycolytic production of adenosine triphosphate (ATP) therefore increasing the muscular capacity for high-intensity tasks ${ }^{8}$. HollidgeHovart, Parolin, Wong, Jones, Heigenhauser ${ }^{5}$ reported that the induced alkalosis seems to enhance the glycogen utilization by the muscle, in addition to a decrease in concentrations of phosphocreatine and accumulation of inorganic phosphate over 15 minutes of cycling in a $75 \%$ maximum oxygen volume (VO2máx) intensity. It is believed that $\mathrm{NaHCO} 3$ intake enhances performance through the lactic acid buffering mechanism produced by exercise $^{3}$.

Studies on the use of $\mathrm{NaHCO} 3$ as an ergogenic resource have been conducted since the 80's for highintensity exercises, however, with a predominance of aerobic characteristics ${ }^{3,4,9,10}$. Specifically, Gledhill ${ }^{4}$ examined $\mathrm{NaHCO} 3$ intake and anaerobic performance and concluded that $\mathrm{NaHCO} 3$ supplementation was shown to be effective in improving runners' performance. Gao, Costill, Horswill ${ }^{3}$ conducted a study comparing the improvement 
of performance in high-intensity exercises and short intervals in a group of 10 trained swimmers, using $\mathrm{NaHCO} 3$ supplementation, placebo (sodium chloride) and a group without supplementation. The authors concluded that the use of NaHCO3 increased the performance of swimmers compared to the other groups analyzed. In addition, Lavander, Bird ${ }^{7}$ demonstrated that trained individuals who underwent a sprint cycle ergometer test presented improvements in performance when previously supplemented with $\mathrm{NaHCO} 3$, with more pronounced differences found after the eighth sprint. However, despite the previous findings, it seems that female cycling athletes respond differently to $\mathrm{NaHCO} 3$ supplementation, with no apparent gains in performance ${ }^{6}$.

Although the relation between $\mathrm{NaHCO} 3$ supplementation and improvement in cyclic (aerobic) highintensity exercise seems to be well established, limited and conflicting experiments focused on the interaction of this supplementation in resistance exercises. The use of $\mathrm{NaHCO} 3$ as a supplement to improve performance in resistance exercises has been gaining support; however, there are not many studies that confirm its effectiveness. For instance, Materko, Simão, Senna, Farinatti ${ }^{11}$ concluded that bicarbonate supplementation had no effect on muscle performance for a 10-RM test with eleven trained men in the pectoral and pull press exercise.

Therefore, further research on the subject is necessary, given the current interest of bodybuilders, fitness enthusiasts, coaches, exercise professionals, and physiologists, to elucidate the effects of $\mathrm{NaHCO} 3$ supplementation in distinct modalities and populations. Thus, the present study aimed to evaluate the impact of NaHCO3 supplementation on repetitions performance, perceived exertion and blood lactate concentration in trained individuals during a resistance exercise session.

\section{Material and Methods}

\section{Participants}

Fourteen trained men participated in this study $(25.14 \pm 3.5$ years; $85.83 \pm 10.18 \mathrm{~kg} ; 1.78 \pm 0.06 \mathrm{~m}$; $26.88 \pm 3.17 \mathrm{~kg} / \mathrm{m}^{2}$ ) with the following inclusion criteria: a) experience in resistance exercise for at least one year, with a frequency of at least three times per week; b) not performing any type of regular physical activity during the study period; c) not having pre-established endocrine diseases, cardiopathies, arterial hypertension, uncontrolled asthma, any musculoskeletal conditions that can act as intervening factors in the practice of the activity (osteoarthritis, recent fracture, tendonitis, and prosthesis use), neurological disorders and use of medications that may cause attention disorders; d) not using any anabolicandrogenic steroids or other ergogenic substances. Before data collection, all participants answered the PAR-Q ques- tionnaire ${ }^{12}$. The subjects read and signed a consent document after being informed of the procedures of the experiment to be carried out during the study, under the Declaration of Helsinki and the resolution of the Brazilian Health Council. This research was approved by the local ethics and research committee under the protocol number: 2.174.170.

\section{Determination of fifteen maximal repetitions (15-RM)}

After two familiarization sessions, the subjects were evaluated for their fifteen maximal repetitions (15-RM) load for two non-consecutive days. On the first day, the 15-RM test was performed for the bench press (BP), machine chest fly (MCF) and triceps pushdown (TR). On the second (non-consecutive) day, the 15-RM retest was conducted in the same exercises, however, with the reverse order to verify the load test reproducibility. During the 15-RM tests, each subject performed 15-RM with a maximum of three trials for each exercise with five minutes of rest interval between attempts ${ }^{13}$. After the determination of the 15-RM for a given exercise, the subjects were allowed to rest for 10 minutes before performing the following 15-RM exercise load test ${ }^{14}$. The highest load lifted during the two days was considered as the load for 15-RM. The n-RM test protocol was previously described $^{13,14}$. Pre-determined technical standards of the selected exercises were followed for each exercise ${ }^{15}$.

Briefly, to minimize errors, the following strategies were adopted: a) standardized instructions on test procedures were offered to participants; b) subjects received instruction on exercise technique; c) body position was held constant; d) verbal encouragement was provided during the testing procedures ${ }^{16}$; e) the mass of all weights and bars used was determined by a precision scale. All tests were performed at the same time of day for the subjects and the warm-up before testing consisted of two sets of 12 repetitions with $40 \%$ of their self-estimated 10-RM $\operatorname{load}^{17,18}$. Subjects were required to utilize a smooth and controlled motion. Pauses were not allowed between the concentric and eccentric phases and the movement had self-selected velocity. No attempt was made to control the movement velocity during each repetition of the exercises $^{19}$. All sessions were supervised by an experienced resistance exercise professional.

\section{Procedures for the determination of Rate Perception Effort (RPE).}

The OMNI Resistance Exercise Scale for an adult was used to obtain the rate of perceived effort (RPE). All subjects performed two familiarization sessions over the course of one week, during this period the participants received the standard OMNI Resistance Exercise Scale instructions and were trained on how to use. The subjects were asked to choose a number on the scale based on their perceived exertion or subjective intensity of exertion, ten- 
sion, discomfort, or fatigue experienced during the exer$\operatorname{cise}^{20}$. The familiarization sessions consisted of the horizontal bench press exercise performed with two sets of 15 repetitions with $40 \%$ of the pre-estimated load with 2 minutes of the interval between sets $^{21}$. During the experimental procedure, immediately after each exercise, subjects were asked to identify their RPE.

\section{Blood lactate}

Blood samples were collected immediately prior, immediately post, 5 minutes post and 15 minutes postexercise. All blood samples were collected from capillary blood. Proper hygiene procedures were performed, as well as the destination of the biological material of the subjects. For the blood collections, surgical disposable latex gloves, cotton, alcohol, and lancets (Softclix) were used. For the blood lactate analysis, reagent strips (Roche, model BMLactate) and a lactometer (Roche, model Accutrend Plus) previously validated ${ }^{22}$ were used.

\section{Nutritional control and supplementation}

Each participant was evaluated during the two days before the exercise sessions to verify possible nutritional interferences that could benefit their performance. A detailed handbook of food weights and volumes and home measures was delivered ${ }^{23}$ so that participants could more accurately report food consumption in household measures. Also, the participants were personally instructed by the researchers regarding the completion of the food register. The analysis of food consumption was qualitative, with the objective of verifying possible food interferences in the tested supplementation. Subjects were instructed not to ingest ergogenic foods, products containing caffeine (energy drinks, soft drinks, coffee, and black tea) and alcohol within 48 hours before testing and experimental sessions.

Participants were instructed to ingest the $\mathrm{NaHCO} 3$ supplementation with a minimum of at least 30 minutes after their last meal, to minimize any possible interference by the remaining food present in the stomach. The subjects performed two training sessions, one with a placebo (sodium chloride) and another with the $\mathrm{NaHCO} 3$ supplementation. It was offered $0.3 \mathrm{~g} / \mathrm{kg} /$ bodyweight of $\mathrm{NaHCO} 3$ and $8 \mathrm{~g}$ of sodium chloride, diluted in $200 \mathrm{ml}$ of filtered water 30 minutes before the start of the experimental sessions. The use of sodium chloride as a placebo was used in previous studies since it reportedly possesses a similar taste to the sodium bicarbonate 7 . To minimize possible differences in gastrointestinal discomfort between all data collections, participants ingested $200 \mathrm{ml}$ of filtered water before the exercise performance.

\section{Procedure}

Forty-eight to seventy-two hours after the final test of 15-RM, the participants were randomly assigned for the interventions that they would participate in the first experimental visit, which were: bicarbonate supplementation (BS) or Placebo. Initially, blood was collected (as previously described) followed by the ingestion of the $\mathrm{NaHCO} 3$ (in BS). All subjects waited 30 minutes before the experimental procedure respecting the physiological timing of the NaHCO3 action. Posteriorly, all subjects completed five sets of a pre-established upper limb exercise session (BP, MCF, and TP) with loads of 15-RM with 1 minute of rest interval between sets. Warm-up before the resistance exercise session consisted of two sets of 12 repetitions with a $40 \%$ load of $15-\mathrm{RM}$ for the BP exercise $^{21}$. It was respected two-minute of rest between warmup and the intervention. Subjects were verbally encouraged to perform five series until voluntary exhaustion ${ }^{16}$, and no attempt was made to control repetition velocity. However, subjects were instructed to use a smooth and controlled movement ${ }^{19}$. The total number of repetitions and the $\mathrm{RPE}^{20}$ were recorded for each set. Blood was collected at specific moments: before (pre), immediately after (post), 5 minutes after (post $5 \mathrm{~min}$ ) and 15 minutes after (post $15 \mathrm{~min}$ ) to the exercise session to determine the subjects' lactate concentration. For all blood withdraws, the participants remained seated in an air-conditioned environment with the temperature being held constant at $20^{\circ} \mathrm{C}$.

\section{Statistical analysis}

The statistical analysis was initially conducted using the Shapiro-Wilk test to verify normality. All variables presented normal distribution. Posteriorly, the interclass correlation coefficient (ICC) was used to determine the reproducibility of the loads between the test and the retest for all exercises. The paired $t$-test was used to verify the total number of repetitions in all sessions and for each exercise between distinct supplementation conditions. The ANOVA for repeated measures was used to test the interactions between the number of repetitions and supplementations condition for repetitions performance and the interactions between blood lactate at the different supplementation conditions and time-points (Pre, post, post 5, and post 15 minutes). If necessary, a Tukey post-hoc test was applied for multiple comparisons. The Friedman test was used to verify the differences in RPE between sets for each exercise. When necessary, a Dunns post-hoc was used for multiple comparisons. The Wilcoxon test was used to compare supplementation conditions.

Also, to determine the magnitude of the findings, the Effect Size (ES; the difference between the pre-test score and the post-test score divided by the standard deviation of the pre-test) was calculated for each exercise set performance, supplementation condition, and blood lactate concentration values. The thresholds proposed by $\mathrm{Cohen}^{24}$ were used to determine the magnitude of the effects. Additionally, the area under the curve (AUC) was calculated by the trapezoidal method for the blood lactate vari- 
able and compared by the paired T-test. The significance level was $p \leq 0.05$. The software used for all statistical analyses was SPSS, version 21.0 (IBM, Inc).

\section{Results}

The ICC data showed an excellent correlation between the 15-RM test and retest for BP $(p=0.96)$, MCF $(p=0.95)$ and TP $(p=0.94)$ exercises. For the total number of repetitions completed during all sessions, no significant difference $(p=0.847)$ between BS $(92.43 \pm 10.43$ reps) vs Placebo (91.71 \pm 8.91 reps) were found. In addition, the ES showed a trivial magnitude in BS this value $(d$ $=0.08)$. For the $\mathrm{BP}(p=0.27)$, MCF $(p=0.09)$ and TR $(p$ $=-0.85)$ no significant difference between the conditions for the total number of repetitions was observed. However, the ES presented a moderate and large difference magnitudes between BS vs Placebo for BP $(d=-0.42)$ and MCF $(d=1.23)$ respectively. These results are represented in Figure 1.

For the BP $(p=0.052)$ and TP $(p=0.933)$ exercises no significant interactions for supplementation conditions and repetition performance for each set were found, in contrast, significant difference was found for this interaction in MCF $(p=0.004)$ exercise. There were significant decreases in repetition performance during subsequent sets for BP $(p<0.001)$ and TP $(p=0.001)$, but there was no decrease observed in MCF $(p=0.365)$. No significant differences were observed in repetition performance between Placebo $v s$ BS (BP $p=0.27$; MCF $p=0.12$; TP $p$ $=0.937)$. Briefly, for the repetition number of each set, decreases in the final sets of the BP and TP compared to the initial set, in both supplementation conditions (Placebo and BS) were found. This reduction in the number of repetitions was maintained even in the initial sets of the second exercise (MCF), which did not lead to significant

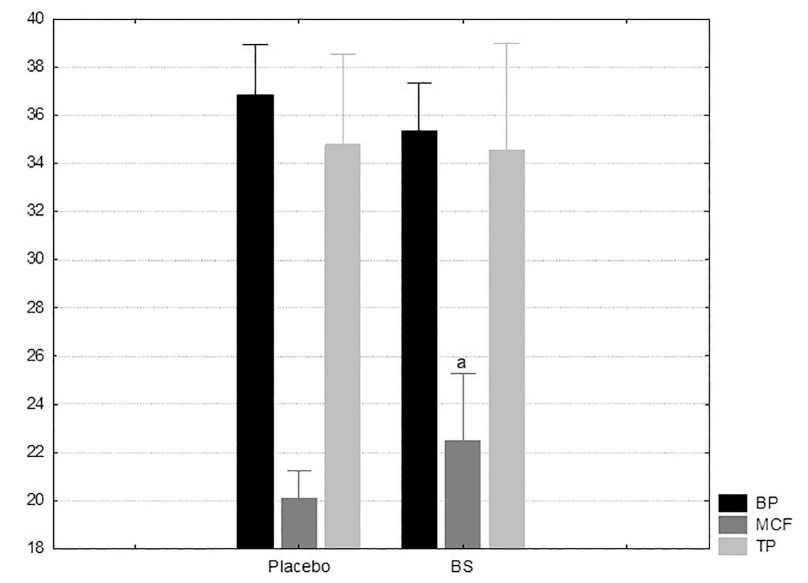

Figure 1 - Total number of repetitions with or without supplementation $($ Data are mean $\pm \mathrm{SD})$. $\mathrm{BP}=$ Bench press; $\mathrm{MCF}=$ Machine chest fly; TP $=$ Triceps pushdown $; \mathrm{BS}=$ Bicarbonate supplementation; ${ }^{\mathrm{a}}$ Large magnitude difference between supplementation condition $(\mathrm{d}>0.8)$. differences. The number of repetitions for each set is represented in Figure 2.

For the blood lactate concentration levels, a significant difference in interactions was observed $(p=$ 0.005 ). Significant differences were found in conditions ( $p$ $=0.016)$ and time point verifications $(p<0.0001)$ main effects. Additionally, the AUC of the BS $(130.3 \pm 26.12)$ vs Placebo $(109.0 \pm 20.43)$ condition presented significantly higher values $(p=0.02)$ and the ES indicated a large magnitude effect for the lactate concentrations in BS condition $(\mathrm{ES}=1.04)$. Data on blood lactate levels are represented in Figure 3.

Both conditions triggered significant increases in RPE values from the third set of BP $(p=0.04)$. For the BS condition, increases in TP were found at the third set, while they appeared only from the fourth set for the Placebo condition. In the MCF exercise, no significant differences were observed in the RPE values for either condition (Table 1).

\section{Discussion}

The most relevant findings of the present study are the non-elevation of the total number of repetitions in the BS condition compared to the Placebo. However, we found a higher overall repetition number in the MCF for BS condition, which possibly demonstrates a greater aiding capacity of the $\mathrm{NaHCO} 3$ supplementation in the single-joint exercise. It seems that it could tolerate a higher magnitude of peripheral fatigue and likely an increased intramuscular metabolic disturbance as previously demonstrated by Rossman, Venturelli, McDaniel, Amann, Richardson $^{25}$. Besides, the order distribution of the performed resistance exercise routine in the experimental session suggests very high fatigue (as observed in RPE values), being widely previously reported ${ }^{26}$. It was also found a higher AUC in the BS condition, which may be related to a more significant number of repetitions in MCF. However, it appears that the buffering action (which is suggested by bicarbonate intake) did not occur.

The $\mathrm{ACSM}^{1}$, on its most recent position stand on nutrition and athletic performance, recognizes the sodium bicarbonate supplementation as an ergogenic resource, with the potential to improve exercise performance of events that would otherwise be limited by acid-base disturbances associated with high rates of anaerobic glycolysis. For instance, high-intensity events of 1-7 minutes; repeated high-intensity sprints; capacity for a high-intensity sprint during endurance exercise. The physiological impact and mechanism of ergogenic effect appear to happen when taken as a pre-exercise acute dose, by promoting the enhancement of the extracellular buffering capacity. An improvement in performance in high-intensity exercise has been demonstrated in several investigations, and its benefits have been associated with 
optimization on acid-base disorders that occur in this type of exercises ${ }^{2-8}$.

A previous experiment conducted by Materko, Novaes, Santos, Veiga ${ }^{11}$ did not present differences in blood lactate concentrations and performance on resistance exercise in subjects who were supplemented with bicarbonate. The study sample was composed of 11 men, aged between 19 and 39 years, and experienced in resis-

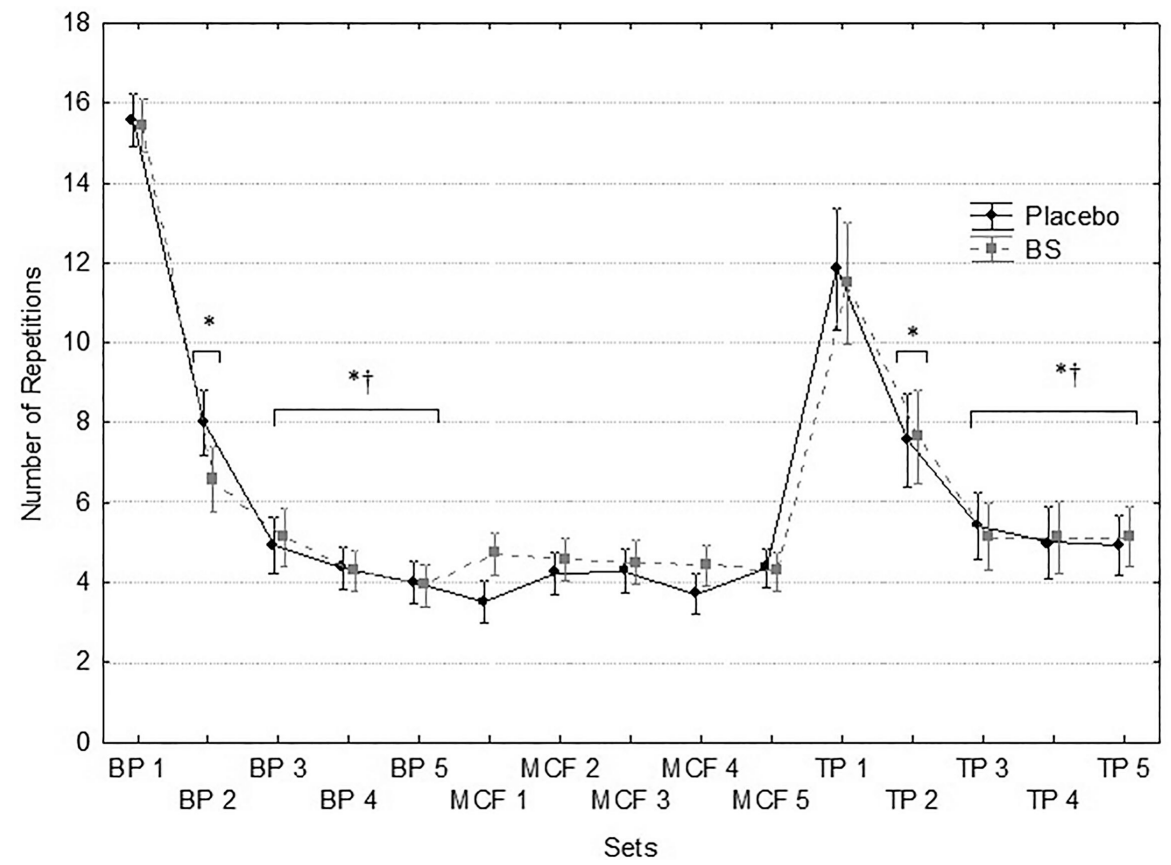

Figure 2 - Number of repetitions each set and exercise with or without supplementation (Data are mean $\pm \mathrm{SD}$ ). $\mathrm{BP}=\mathrm{Bench}$ press; $\mathrm{MCF}=\mathrm{Machine}$ chest fly; $\mathrm{TP}=$ Triceps pushdown ; BS = Bicarbonate supplementation; * Significant difference to the Pre $(\mathrm{p} \leq 0.05)$.; $\dagger$ Significant difference to the Post $(\mathrm{p} \leq$ $0.05)$.

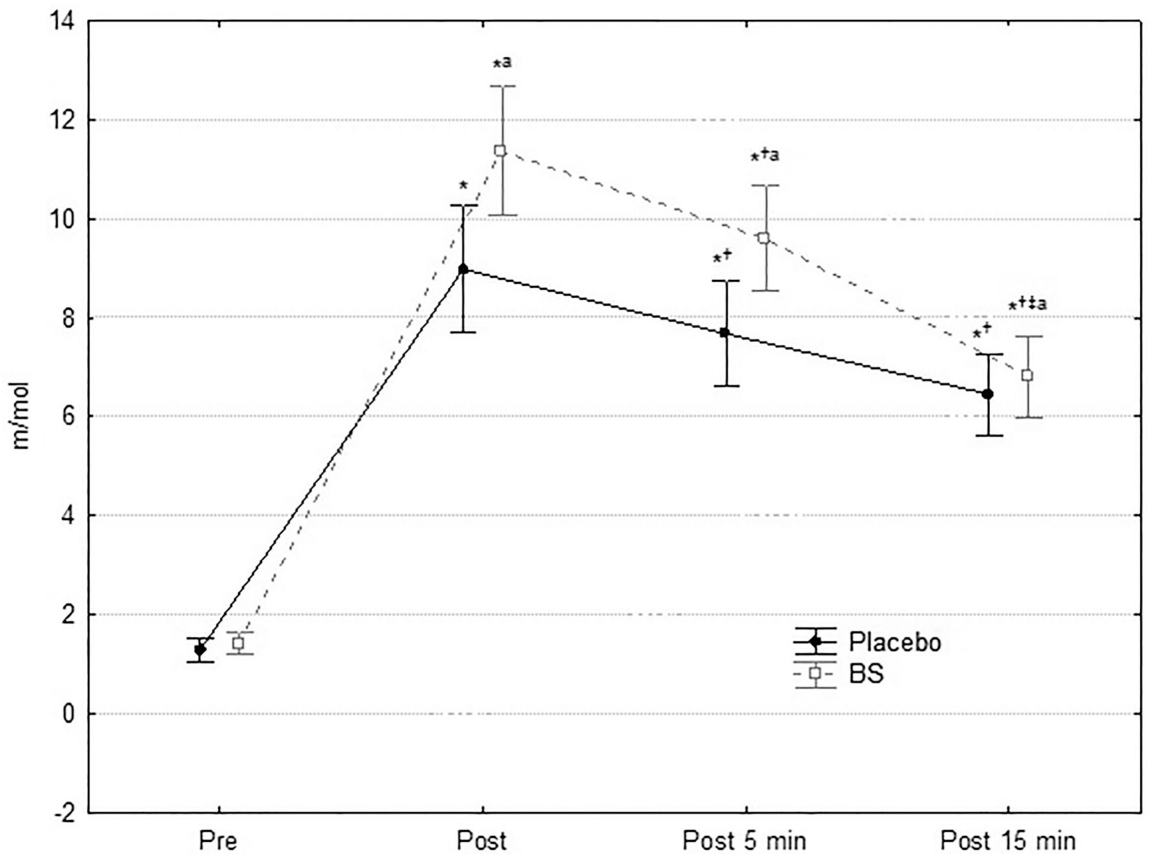

Figure 3 - Lactate concentrations at pre-exercise (Pre), and immediately (Post), 5 minutes (Post 5 min), and 15 minutes (Post 15 min) after completing three upper body exercises in different supplementation condition (Data are mean $\pm \mathrm{SD}$ ). * Significant difference to the Pre $(\mathrm{p} \leq 0.05)$.; $\dagger$ Significant difference to the Post $(\mathrm{p} \leq 0.05)$.; $¥$ Significant difference to the Post $5 \min (\mathrm{p} \leq 0.05)$.; ${ }^{\mathrm{a}}$ Significant difference to BS condition $(\mathrm{p} \leq 0.05)$. 
Table 1 - Rating of perceived exertion (RPE) values for each set on both supplementation conditions (Data are median [25\%-75\% of frequency]).

\begin{tabular}{|c|c|c|c|c|c|}
\hline & Set 1 & Set 2 & Set 3 & Set 4 & Set 5 \\
\hline \multicolumn{6}{|l|}{ BS } \\
\hline $\mathrm{BP}$ & $4.25(2-6)$ & $5(3-6.5)$ & $5.5(4-7)^{*}$ & $5.5(4-7)^{* \dagger}$ & $6.25(4-7.5)^{* \dagger}$ \\
\hline MCF & $5.25(4-6.25)$ & $5.5(4-7.25)$ & $5.75(4-7.25)$ & $6(4-7.25)$ & $6(4-7.5)$ \\
\hline $\mathrm{TP}$ & $6.5(5-8)$ & $7(6.25-8)$ & $7.5(6-8.5)^{*}$ & $7.5(6.75-8.5)^{* \dagger}$ & $8(7.5-9)^{* \dagger}$ \\
\hline \multicolumn{6}{|c|}{ Placebo } \\
\hline BP & $4.25(3-6)$ & $5.5(4-6.25)$ & $6.5(5-7)^{* a}$ & $6.5(5.5-7.25)^{* \dagger}$ & $6.5(6-7.75)^{* \dagger}$ \\
\hline $\mathrm{MCF}$ & $5.5(5-7.2)$ & $6.25(5-6.5)$ & $6.75(5.75-7)$ & $7(5.75-7)$ & $7(6-7.5)$ \\
\hline $\mathrm{TP}$ & $7(5.5-8)$ & $7.5(6.5-8)$ & $8(6.25-8)$ & $8(6.25-8.5)^{*}$ & $8.25(7-9)^{* \dagger}$ \\
\hline
\end{tabular}

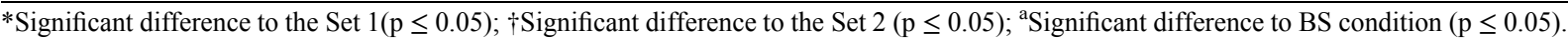

tance exercise. Subjects were randomly assigned to ingest two different solutions, one of either sodium bicarbonate $(0.3 \mathrm{~g} / \mathrm{kg})$ or sodium chloride $(0.045 \mathrm{~g} / \mathrm{kg})$ before the strength assessment. Each subject performed 10-RM on the BP and pull press following these three routines: without supplementation; with sodium bicarbonate supplementation; with sodium chloride supplementation. No significant difference was observed between the evaluated procedures, concluding that sodium bicarbonate supplementation did not affect the BP strength performance. Despite the fact that different methods, intensities, volumes, and exercises, performed by Materko, Novaes, Santos, Veiga ${ }^{11}$, both studies did not show improvement in the performance of individuals when supplemented with sodium bicarbonate. However, the present study showed an increase in the MCF exercise performance, due the fatigue associated with the high volume and intensity of the training was higher when compared to the study of Materko, Materko, Novaes, Santos, Veiga ${ }^{11}$.

Nevertheless, depending on the bioenergetic demand and the characteristics of the exercise, the results might be different $^{2-8}$. Specifically, Gao, Costill, Horswill ${ }^{3}$ analyzed the effects of bicarbonate supplementation on exercise performance in ten male swimmers during five different interventions. Each intervention consisted of five sprints of 90 meters, of the swim crawl, with an interval of two minutes between the sprints. The protocols included two bicarbonate supplements, two of placebo and one intervention without supplementation. No significant difference was found in performance or blood measurements between the placebo group and the non-supplementation group. However, the group supplemented with bicarbonate showed a substantial improvement in performance in the fourth and fifth sprints. They concluded, therefore, that pre-exercise bicarbonate supplementation is capable of improving performance, possibly by facilitating the efflux of hydrogen ions from the muscle and consequently retarding fatigue. In our study, likely due to the specificity presented in the resistance exercise, it was not possible to observe results in performance improvement, only in the exercise of the MCF, differing from the study above, being more elucidated the ergogenic effect of this supplementation in activities with aerobic characteristics.

Lavender, Bird ${ }^{7}$ made a study with the purpose of assessing the effect of sodium bicarbonate ingestion upon repeated bouts of intensive short-duration exercise. Twenty-three subjects participated in the investigation. Subjects completed six trials; three following the ingestion of sodium bicarbonate $(300 \mathrm{mg} / \mathrm{kg}$ body weight $)$ and three following the ingestion of a placebo ( $8 \mathrm{~g}$ sodium chloride). Each trial consisted of ten ten-second sprints on a cycle ergometer with a 50-second recovery between each sprint. 'Peak power' and 'average power output' during each tensecond sprint was measured. The authors concluded that during exercise consisting of repeated, short-duration sprints, power output was enhanced following the ingestion of sodium bicarbonate.

Senna, Scudese, Carneiro, Torres, Queiroz, Dantas ${ }^{27}$ demonstrated in their study that the performance during the repetitions can be significantly affected and present a reduction in the number of repetitions performed when the interval between the series is smaller, in multi and singlejoint exercises. In another study by Senna, Rodrigues, Sandy, Scudese, Bianco, Dantas ${ }^{28}$, it was observed that the smaller the interval between the set, the greater the reduction in performance, being evident in single-joint exercises the need for a recovery of more than 3 minutes, for maintenance of the same. In this sense, previous study compared the influence of different manipulations of the methodological variable of intervals between the series in the resistance exercise for single and multi-joint exercises on performance, RPE and blood lactate ${ }^{29}$. The participants (twelve trained men) performed four different training sessions (two with the BP and the other with the MCF). In each session, five 10-RM sets were performed until fatigue with a 1- or 3-minute rest interval in a counterbalanced cross-over experiment. The longer interval promoted a greater total number of repetitions in both exercises. As for RPE, progressive elevations occurred after the third grade in all conditions tested. For the blood lactate concentration, the differences between the intervals occurred in the pectoral fin with higher elevations presented in the 
shorter intervals. These data allow the assertion that changes in the volume of the single-joint (MCF) exercise can potentiate blood lactate responses, as occurred in the present experiment. Therefore, it is believed that sodium bicarbonate supplementation was effective in delaying exercise fatigue since it had a higher number of repetitions in the supplementation group.

The values of RPE were used to evaluate the relative intensity of resistance exercise, as previously suggested by Lagally and Robertson ${ }^{20}$. In both experimental protocols, the methodological interval between set and exercises (1 minute) may have emphasized anaerobic glycolysis to a greater extent to compensate for the incomplete resynthesize of phosphocreatine ${ }^{29}$. The more significant emphasis on anaerobic glycolysis is associated with $\mathrm{H}+$ accumulation, as well as decreases in intracellular fluid $\mathrm{pH}$. The result of the afferent response of chemoreceptors and nociceptors may increase $\mathrm{RPE}^{20}$. Previous studies with 10-RM intensities ${ }^{14,19,27,29}$ demonstrated increases in RPE during consecutive sets, and these increases were more evident at shorter intervals ( 1 minute) when compared to longer intervals long ( 3 and 5 minutes). More recently, it was observed significant increases in RPE at 1-minute intervals from the third set of single and multi-joint exercises with a 3-RM intensity ${ }^{21}$. These results suggest a better perception of fatigue in shorter when compared with larger rest periods, independently of the exercise modality (single or multi-joint). In the presented results, high values of RPE are observed, suggesting that during all the sets significant fatigue occurred, raising the levels of blood lactate, mainly after the third sets of bench chest, regardless of supplementation conditions.

This experiment adds to the growing body of knowledge related to sodium bicarbonate supplementation and its effects on resistance exercise performance and information on blood lactate and RPE responses.

\section{Conclusion}

We conclude that sodium bicarbonate supplementation was not able to increase performance during resistance exercise (except for single-joint exercise performed at high perceived exertion), nor did it alter blood lactate levels during and after training. With these data, it is possible to extrapolate that the use of bicarbonate before resistance exercise should be carefully performed in extremely long exercise routines (with several multi-joint exercises for the same muscle group) and short intervals to increase performance. These data can be of high relevance given possibility of low cost in different fields of action of the health professionals, either now not recommending the use of this supplement because they perceive training that does not generate great fatigues, sometimes, as in the sports sphere, noting the need for small performance gains. Besides, we strongly recommend different investi- gations on this topic so that the actual interaction between the supplementation and the possible benefits in the resistance exercise can be clarified.

\section{References}

1. American College of Sports Medicine. Position stand on nutrition and athletic performance. Med Sci Sports Exerc. 2015; 48 (3): 543-568. Doi: 10.1249/MSS.0000000000000852

2. Carr AJ, Hopkins WG, Gore CJ. Effects of acute alkalosis and acidosis on perfoemance. Sports Med. 2011; 41(10): 801-814. Doi: 10.2165/11591440-000000000-00000

3. Gao J, Costill DL, Horswill CA. Sodium bicarbonate ingestion improves performance in interval swimming. Eur $\mathrm{J}$ Appl Physiol. 1988; 58: 171-174.

4. Gledhill N. Bicarbonate ingestion and anaerobic perfomance. Sports Med. 1984; 1: 177-180. Doi: 10.2165/ 00007256-198401030-00001

5. Hollidge-Hovart MG, Parolin ML, Wong D, Jones NL, Heigenhauser GJ. Effect of induced metabolic alkalosis on human skeletal muscle metabolism during exercise. Am J Physiol Endocrinol Metab. 2000; 278: E316-E329. Doi: 10.1152/ajpendo.2000.278.2.E316

6. Kozak-Collins K, Burke ER, Schoene R. Sodium bicarbonate ingestion does not improve performance in women cyclists. Med Sci Sports Exerc. 1994; 26 (12): 1510-1515.

7. Lavander G, Bird SR. Effect of sodium bicarbonate ingestion upon repeated sprints. Br. J. Sports Med. 1989; 23: 4145. Doi: $10.1136 / \mathrm{bjsm} .23 .1 .41$

8. Raymer GH, Marsh D, Kowalchuck JM, Thompson RT. Metabolic effects of induced alkalosis during progressive forearm exercise to fatigue. J Appl Physiol. 2004; 96: 2050-2056. Doi: 10.1152/japplphysiol.01261.2003

9. Matson LG, Tran ZV. Effects of sodium bicarbonate ingestion on anaerobic performance: a meta-analytic review. Int J Sport Nutri Exerc Metab. 1993; 3: 2-28. Doi: 10.1123/ ijsn.3.1.2

10. Mcnaughton L, Cedaro R. The effect of sodium bicarbonate on rowing ergometer performance in elite rowers. Aust J Sci Med Sport. 1991; 23: 66-69.

11. Materko W, Novaes JS, Santos EL, Veiga GV. Effect of bicarbonate supplementation on the muscular strength. JEP online. 2008; 11: 25-33. Doi: 10.1249/00005768200605001-01723

12. Shephard RJ. PAR-Q, Canadian home fitness test and exercise screening alternatives. Sports Med. 1988 5: 185-195. Doi: 10.2165/00007256-198805030-00005

13. Polito MD, Simão R, Senna GW, Farinatti PTV. Efeito hipotensivo do exercício de força realizado em intensidades diferentes e mesmo volume de trabalho. Rev Bras Med Esporte. 2003. 9: 69-73. http://www.scielo.br/pdf/rbme/ v9n2/v9n2a03.pdf

14. Senna G, De Salles BF, Prestes J, Mello RA, Simão R. Influence of two different rest interval lengths in resistance training sessions for upper and lower body. J Sports Sci Med. 2009; 8: 197-202. https://www.ncbi.nlm.nih.gov/pmc/ articles/PMC3761475/pdf/jssm-08-197.pdf 
15. Baechle TR, Earle RW. Essentials of strength training and conditioning. $2^{\text {nd }}$ edition: Human Kinetics Champaign, 2000.

16. McNair PJ, Depledge J, Brettkelly M, Stanley SN. Verbal encouragement: Effect on maximum effort voluntary muscle action. Br J Sports Med. 1996; 30: 243-245. Doi: http:// dx.doi.org/10.1136/bjsm.30.3.243

17. Scudese E, Willardson JM, Simão R, Senna G, De Salles $\mathrm{BF}$, Miranda H. The effect of rest interval length on repetition consistency and perceived exertion during near maximal loaded bench press sets. J. Strength Cond. Res. 2015; 29: 3079-3083. Doi: 10.1097/JSC.0000000000000214

18. Scudese E, Simão R, Senna G, Vingren JL, Willardson JM, Baffi M, Miranda, H. Long rest interval promotes durable testosterone responses in high-intensity bench press. J. Strength Cond. Res. 2016; 30: 1275-1286. Doi: 10.1519/ JSC.0000000000001237

19. Senna G, Willardson JM, De Salles BF, Scudese E, Carneiro F, Palma A, Simão R. The effect of rest interval length on multi- and single-joint exercise performance and perceived exertion. J. Strength Cond. Res. 2011; 25: 57-62. Doi: 10.1519/JSC.0b013e318212e23b

20. Lagally KM, Robertson RJ. Construct validity of the OMNI resistance exercise scale. J. Strength Cond. Res. 2006; 20: 252-256. Doi: 10.1519/R-17224.1

21. Senna GW, Willardson JM, Scudese E, Simão R, Queiroz C, Avelar R, Dantas EHM. Effect of different interset rest intervals on performance of single and multijoint exercises with near-maximal loads. J. Strength Cond. Res. 2016; 30: 710-716. Doi: 10.1519/JSC.0000000000001142

22. Baldari C., Bonavolontà V, Emerenziani GP, Gallotta MC, Silva AJ, Guidetti L. Acurracy, reliability, linearity of Accutrend and Lactate Pro versus EBIO plus analyzer. Eur J Appl Physiol. 2009; 107, 105-111. Doi: 10.1007/s00421009-1107-5

23. Vitolo M R. Nutrição da gestação ao envelhecimento. $2 .^{\text {ed }}$. RUBIO, 2008.

24. Cohen J. Statistical Power Analysis for the Behavioral Sciences. $2^{\text {nd }}$ edition: Lawrence Erlbaum Hillsdale, 1988.
25. Rossman MJ, Venturelli M, McDaniel J, Amann M, Richardson RS. Muscle mass and peripheral fatigue: a potential role afferent feedback? Acta Physiol, 2012; 206: 242-250. Doi: 10.1111/j.1748-1716.2012.02471.x

26. Simão R, Salles BF, Figuereido T, Dias I, Willardson JM. Exercise order in resistence training. Sport Med. 2012. 42: 1-15. Doi: 10.2165/11597240-000000000-00000

27. Senna G, Scudese E, Carneiro F, Torres J, Queiroz C, Dantas E. Multi-joint and single-joint exercise performance and perceived exertion with several different recoveries. JEPonline. 2015; 18: 91-100.

28. Senna GW, Rodrigues BM, Sandy D, Scudese E, Bianco A, Dantas EHM. Heavy vs light load single-joint exercise performance with different rest intervals. J Hum Kinet. 2017; 58: 197-206. Doi: 10.1515/hukin-2017-0077

29. Senna GW, Figueiredo T, Scudese E, Baffi M, Carneiro F, Moraes E, Miranda H, Simão R. Influence of different rest interval length in multi-joint and single-joint exercises on repetition performance, perceived exertion, and blood lactate. JEPonline. 2012; 15: 96-106.

\section{Corresponding author}

Carlos José Nogueira Laboratory of Biosciences of Human Motricity, (LABIMH), Federal University of State of Rio de Janeiro, (UNIRIO), Rio de Janeiro, Brazi.

E-mail: carlosjn29@yahoo.com.br

Manuscript received on November 25, 2019

Manuscript accepted on January 10, 2020

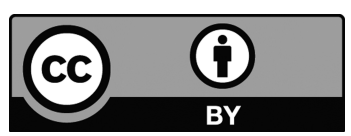

Motriz. The Journal of Physical Education. UNESP. Rio Claro, SP, Brazil - eISSN: 1980-6574 - under a license Creative Commons - Version 4.0 